\title{
Atomic-Scale Electron-Beam Sculpting of Defect-Free Graphene Nanostructures
}

\author{
Bo Song, Grégory F. Schneider, Qiang Xu, Grégory Pandraud, Cees Dekker, Henny Zandbergen
}

Kavli Institute of Nanoscience, Delft University of Technology, Lorentzweg 1, 2628 CJ Delft, The Netherlands

First isolated in $2004,{ }^{1}$ graphene has received tremendous scientific attention due to its unique electronic properties. ${ }^{2}$ Graphene also features edge dynamics ${ }^{3}$ and mechanical properties, ${ }^{4}$ opening up even more opportunities such as its use to sequence genomic DNA using nanopores. ${ }^{5}$ In order to harvest the many promising properties of graphene in applications, a technique is required to cut, shape or sculpt the material on a nanoscale without damage to its atomic structure, as this drastically influences the electronic properties of the nanostructure. Here, we reveal a temperature-dependent self-repair mechanism allowing damage-free atomic-scale sculpting of graphene using a focused electron beam. Our technique allows reproducible fabrication and simultaneous imaging of singlecrystalline free-standing nanoribbons, nanotubes, nanopores, and single carbon chains.

Temperature has a remarkable effect on the changes induced by $300 \mathrm{keV}$ electrons (Figure 1). At room temperature (RT), a rapid amorphisation occurs, which prevents detailed high-resolution electron microscopy (HREM). At temperatures of $200^{\circ} \mathrm{C}$, we observe that electron beam irradiation leads to amorphisation with only short range order (Figure 1a). At $500^{\circ} \mathrm{C}$, the electron beam results in the formation of polycrystalline monolayers. The single crystalline graphene transforms into polycrystalline graphene with clear straight but short grain boundaries. At $700{ }^{\circ} \mathrm{C}$, remarkably, graphene conserves its full crystallinity even under a very intense electron beam, as shown in Figure 1c. Figure 2 shows various graphene nanostructures made at $600^{\circ} \mathrm{C} \sim 700^{\circ} \mathrm{C}$. Carbon nanotubes can be made using elongated electron beams with a shape similar to the holes made (Figure 2a, inset). Figure 2(b) shows a flat single-walled nanotube (double layer graphene nanoribbon) made from few layers graphene. The inset gives the inferred tube shape with the HREM image averaged by translation, and the observed (red) and estimated (green) hexagon positions for a flat tube. Blue dots in the side view represent individual carbon atoms. Figure 2 (c) shows nanoribbon made from single layer graphene, where the monolayer was first made from few layers graphene. Note that the edges of the nanoribbon are armchair, as are most of the edges of the two holes. Inset shows the image simulation of two carbon ad-atoms attached to the graphene armchair edges and pointed out with red arrows. Figure 2 (d)-(e) showed a double carbon chain and a single carbon chain (formed from the double chain), respectively.

References

[1] K.S. Novoselov et al, Science, 306 (2004) 666.

[2] A.H. Castro Neto et al, Rev. Mod. Phys., 81 (2009) 109.

[3] C. O. Girit et al, Science, 323 (2009) 1705.

[4] C Lee et al, Science, 321 (2008) 385.

[5] G. F. Schneider et al, Nano Lett., 10 (2010) 1912.

[6] This work was supported by Nano-IMaging under Industrial Conditions (NIMIC) and funding from the European Union's Seventh Framework Programme (FP7/2007-2013) under grant agreement $n^{\circ} 201418$ (READNA). 

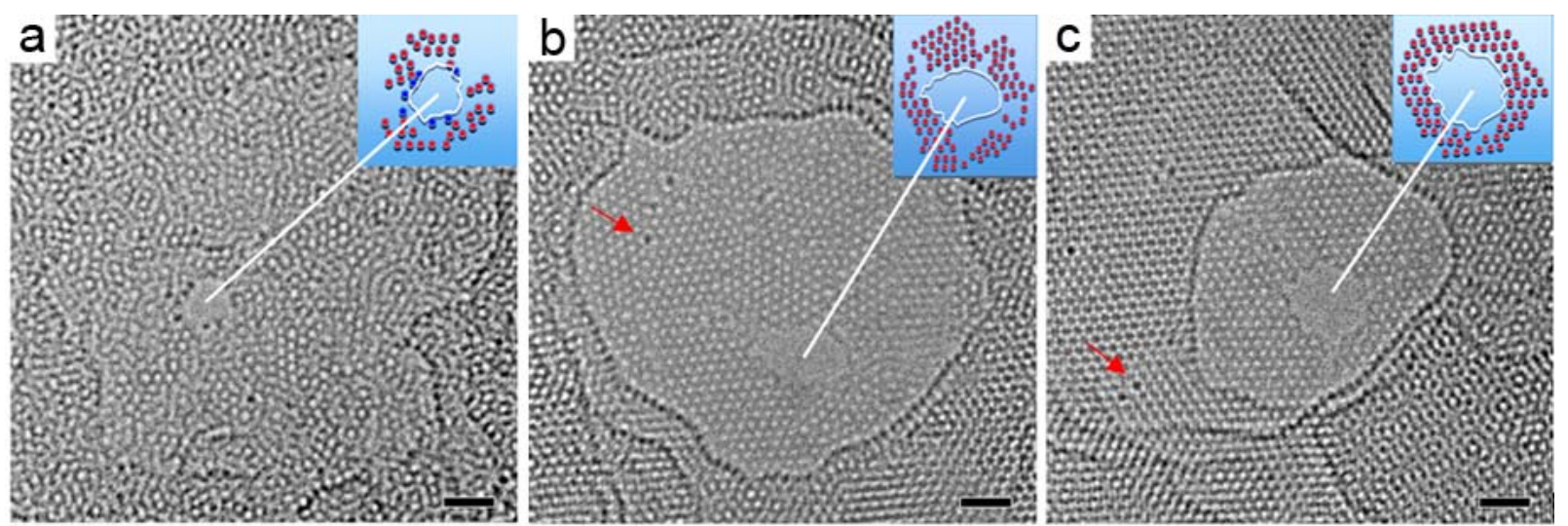

Figure 1. Influence of temperature on the sculpting of few-layer graphene by an electron beam. (a), at $200{ }^{\circ} \mathrm{C}$. (b), at $500{ }^{\circ} \mathrm{C}$. (c), at $700{ }^{\circ} \mathrm{C}$. Red arrows indicate some of the $\mathrm{C}$ ad-atoms trapped at defects. The insets in (a)-(c) show the positions of the identifiable hexagons (red dots) and the estimated position of the edge (white line). The blue dots in the inset at $200{ }^{\circ} \mathrm{C}$ are ad-atoms. Scale bars, $1 \mathrm{~nm}$.

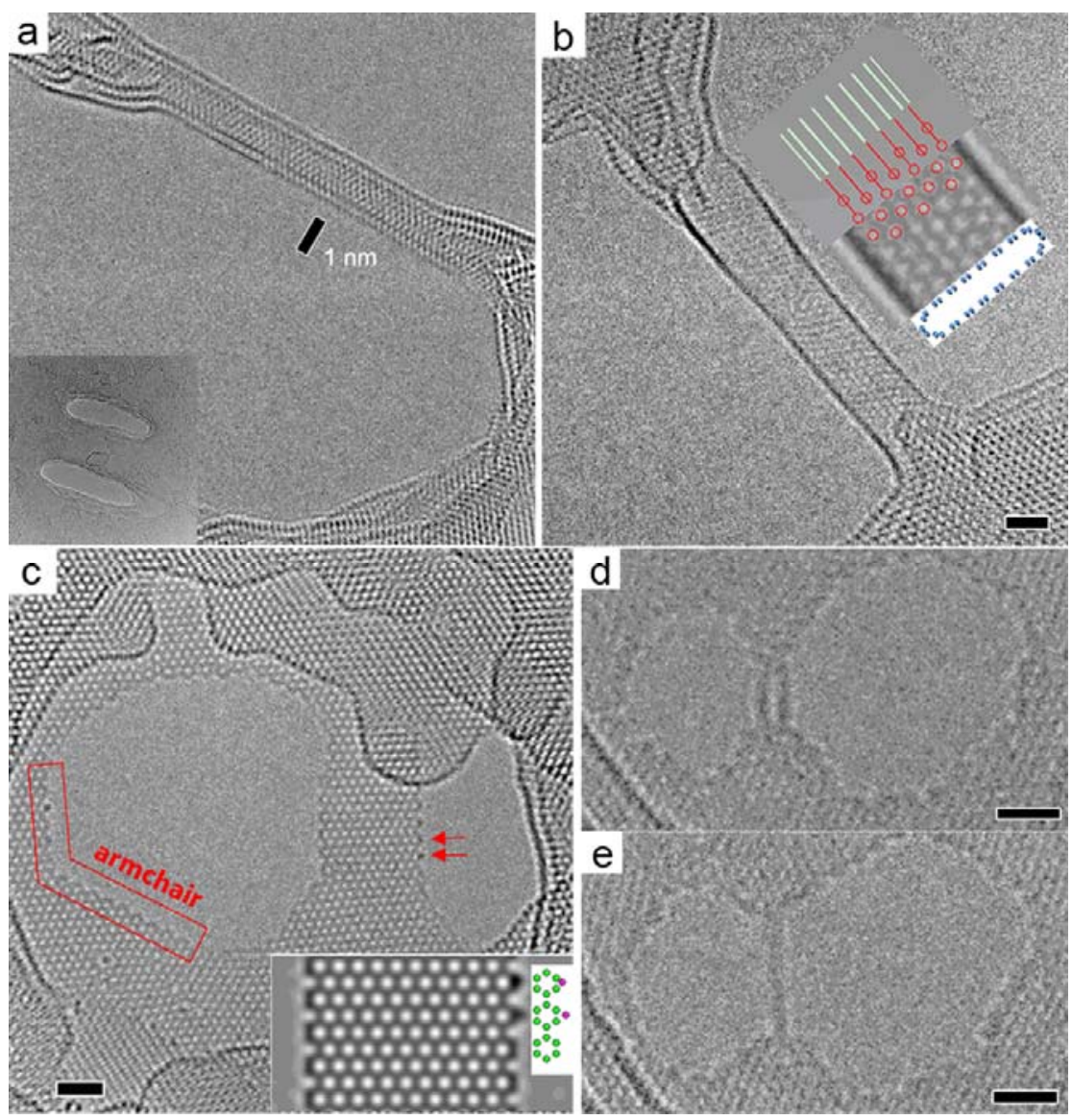

Figure 2. Variety of crystalline carbon nanostructures made with $300 \mathrm{kV}$ electrons at $600-700{ }^{\circ} \mathrm{C}$. (a) double wall carbon nanotube. (b) flat single wall carbon nanotube (double layer graphene nanoribbon). (c) armchair graphene nanoribbon. (d)-(e) carbon chains. Scale bars, $1 \mathrm{~nm}$. 